\title{
Operational semantics for product-form solution
}

\author{
Maria Grazia Vigliotti \\ Department of Computing, Imperial College London, \\ 180 Queen's Gate, London SW7 2BZ, UK \\ maria.vigliotti@imperial.ac.uk
}

\begin{abstract}
In this paper we present product-form solutions from the point of view of stochastic process algebra. In previous work [16] we have shown how to derive product-form solutions for a formalism called Labelled Markov Automata (LMA). LMA are very useful as their relation with the Continuous Time Markov Chains is very direct. The disadvantage of using LMA is that the proofs of properties are cumbersome. In fact, in LMA it is not possible to use the inductive structure of the language in a proof. In this paper we consider a simple stochastic process algebra that has the great advantage of simplifying the proofs. This simple language has been inspired by PEPA [10, however, detailed analysis of the semantics of cooperation will show the differences between the two formalisms. It will also be shown that the semantics of the cooperation in process algebra influences the correctness of the derivation of the product-form solutions.
\end{abstract}

\section{Introduction}

In this paper we present product-form solutions from the point of view of stochastic process algebra. The main motivation for this work is twofold: on one side, our formalisation clarifies the basic mechanisms that govern product-form solutions in Continuous Time Markov Chains (CTMCs), on the other side, we can generalise the notion of product-form solutions beyond queuing theory. Product-form solutions are efficient solutions for stationary distributions in CTMCs in general, while so far product-form solutions have been studied mostly in the area of performance evaluation/ queuing theory. The work presented here is an extension of previous work [16] where we have shown how to derive product-form solutions for a formalism called Labelled Markov Automata (LMA). In very simple terms, LMA describe the state space of CTMCs as labelled graph decorated with transition rates. LMA are equipped with a basic mechanism to build complex CTMCs. LMA have proved very useful in helping to understand basic mechanisms that govern product-form solutions, and in providing a very elegant proof of the theorem GRCAT [16]. However, the disadvantage in using LMA is that the proofs, even for simple properties, are cumbersome. In LMA it is not possible to use the inductive structure of the language in a proof.

In this paper we improve on previous work [16] by considering a simple stochastic process algebra (SSPA), which preserves the semantics of cooperation 
of LMA. This simple language has been inspired by PEPA [10, however detailed analysis of the semantics of cooperation will show the differences between the two formalisms.

In this paper we investigate the general principle that determines productform solutions in CTMCs, and we show that the semantics of cooperation is crucial for the correct derivation of product-form solutions. We will introduce a simple language equipped with a rather unique, and possibly counterintuitive semantics, which guarantees the existence of product-form solutions. We will argue that the semantics presented here, is precisely what is needed to model rigorously product-form solutions for CTMCs. We shall also consider a biological example to show an interesting application of product-form solutions to a context different from queuing theory.

\section{Related work}

There is vast literature on the topic of product-form solutions and process algebra, and on the formalisation of the intrinsic mechanisms that determine product-form solution $[19|12| 8|2| 9|7 / 2| 3$. On the relationship between process algebra and product-form solutions, Hillston, Thomas and Clark, played a major role [13 12 8 11/6 19. The common denominator in these papers is the use of PEPA to model processes that are known to enjoy product-solution, and to extract, via PEPA, the modular properties of such processes. This body of work has demonstrated to the community the modelling power of PEPA. It was shown in [8] that quasi-reversible structures can be modelled in PEPA, together with a large variety of product-from solutions. We differ from the work carried out in PEPA, as our goal is not to define 'yet another stochastic process algebra' to model product-form solutions, but to design a language and a semantics to describe only the CTMCs that enjoy product-form solutions. With our formalism it is relatively easy to find new product-form solutions for CTMCs. This was not achieved in previous work.

Another formalism that has been extensively used is the Generalised Stochastic Petri Nets (GSPN) 2/7/2/3- to cite a few articles. The emphasis is to understand which GSPN enjoy product-form solutions, and what conditions on GSPN are necessary to yield product-form solution [7. We differ from the work on the GSPN as we use process algebra, and also because of the generality of our results. In this paper, and in previous work, the effort has been directed in defining a set of sufficient conditions that guarantee product-form solutions for time-homogenous CTMCs. Finally, it must be mentioned that similar efforts have been carried out by the community in performance $14|5| 18$.

The class of product-form solutions considered by [18 is rather limited, while a true advancement was made by [14]5] with the notions of quasi-reversibility. Quasi-reversibility was introduced by Kelly [14, and used only on the context of queuing networks. In [5], great efforts were successfully made to show generality and robustness of quasi-reversibility. Nearly all product-form solutions known in queueing networks are derived using quasi-reversibility. It was proved in [5] that 
quasi-reversibility is a sufficient condition for product-form solutions. The conditions of Theorem 1 can be seen as formalisation of quasi-reversibility. The main difference between Theorem 1 and quasi-reversibility lies in the formalisation of the 'connection' of CTMCs. The way in which queues are connected together is expressed in natural language [5]. This is the main weakness of the work. Since it is not clear how to 'connect' CTMCs together, only networks of queues are considered. Understanding of how to connect queues together is clear in the community of performance evaluation. Our work goes further as it specifies, in a rigorous way, the 'connection' or, better, the cooperation among CTMCs. Note that we have only sufficient conditions for product-form solutions, not necessary conditions. As consequence, there are product-form solutions that we cannot characterise, for example [4. We leave for future work formal development to deal with such product-form solutions.

\section{A simple language}

In this section we introduce a simple stochastic process algebra, SSPA. The main motivation to introduce such a formalism is to verify that the conditions for product-form solutions can be modelled in a language. SSPA is defined in a rather unusual way, but follows in the spirit ideas that were discussed in 1312. We initially define simple processes. These are composed essentially by choice and by recursion.Similarly to PEPA, simple processes may or may not characterise a CTMC. Some simple process are incomplete, according to PEPA terminology, in the sense that some transitions lack the information about the rate. Such information can be inserted via a new operator: the closure. A second layer of processes is defined, as cooperation of simple processes. The operator for cooperation has been inspired by PEPA, but differently form PEPA is an n-nary -operator, like choice. Such operator, differently form PEPA-bow, cannot be expressed as multiple composition of the binary association.

To formally define the language we assume the existence of a set of variables Var, and a set of actions Act on which the letter $a, b, c \ldots$ range over it.

Definition 1 (Simple processes). The set of simple process, $\mathcal{P}$, is is given by the following syntax:

$$
\begin{aligned}
& \mathrm{E}::=0 \mid \mathrm{D} \\
& \mathrm{M}::=\sum_{i \in I}\left(a_{i}, r_{i}\right) . \mathrm{E}_{i} \mid \mathrm{M}_{[a \leftarrow \lambda]}
\end{aligned}
$$

where $I$ is a finite set of indexes.

For clarity in the notation we use the greek letters $\lambda, \mu, \ldots$ range over the set of positive real numbers $\mathbb{R}^{+}$, the letters $x, y, x, \ldots$ range over Var and the letter $r$ ranges over $\mathbb{R}^{+} \cup$ Var. When writing a variable in processes, we use a subscript that refers to the label. For example we would write $\left(a, y_{a}\right)$.E but not $\left(a, y_{b}\right)$.E. A simple processes stand for the building blocks which are ultimately used to compose complex CTMCs. Nil, written 0, is the empty process; D is the symbol for the identifier. Identifiers are equipped with identifier equations such 
as $\mathrm{D} \stackrel{d f}{=} \mathrm{M}$. The choice, $\sum_{i \in I}(a, r) . \mathrm{E}_{i}$, represents the standard selection of one of the possible transitions, and finally there is a new operator closure $\mathrm{M}_{[a \leftarrow \lambda]}$. This operator transforms all transitions labelled with the pair $a$ and a variable into transitions labelled with the pair $a$ and the real number $\lambda$. The role of closure will become clear in the later development of the paper. The grammar of simple process aims to define the transition graph of a labelled CTMC, but not all transition graphs derived from this grammar are CTMCs due to the presence of variables. Examples of this kind can be seen in Fig. 1.

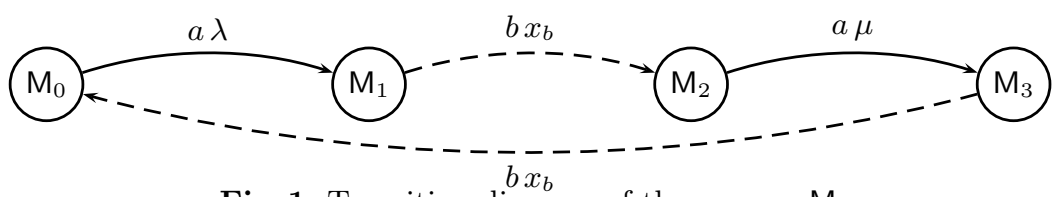

Fig. 1. Transition diagram of the process $M_{0}$

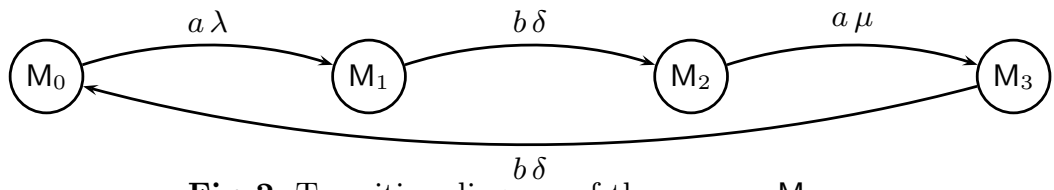

Fig. 2. Transition diagram of the process $\mathrm{M}_{0[b \leftarrow \delta]}$

Informally, we can say that the closure operator would transform a transition graph of the simple process $\mathrm{M}_{0} \stackrel{d f}{=}(a, \lambda) \cdot\left(b, x_{b}\right) \cdot(a, \mu) \cdot\left(b, x_{b}\right) \cdot \mathrm{M}_{0}$ as in Fig. 1 as one in Fig 2 for the simple process $\mathrm{M}_{0[b \leftarrow \delta]}$.

If all transitions of a process are decorated with a real number, as in in Fig. 2 then the underlying model description is a time-homogenous CTMC, similar to PEPA. To formally define how to derive the CTMC of a given simple process, we need to give a formal semantics to SSPA via labelled transition system.

Definition 2. A labelled transition system for simple processes written $\rightarrow: \mathcal{P} \times$ $\left(\right.$ Act $\times \mathbb{R}^{+} \cup$ Var $) \times \mathcal{P}$ is the smallest multi-relation that satisfies the rules in Table 1 .

We write $\mathrm{M} \stackrel{a, r}{\longrightarrow} \mathrm{M}^{\prime}$ if $\left(\mathrm{M},(a, r), \mathrm{M}^{\prime}\right) \in \rightarrow$, and $\rightarrow^{*}$ for the transitive closure of $\rightarrow$.

In what follows, we consider a relation that is generally defined in $\pi$-calculus 17] structural congruence. Structural congruence is a relation preserved by all operators of the calculus, i.e a congruence, that identifies terms that should not be distinguished for semantical reasons. Hillston [10] defined a similar relation in a operational way as isomorphism.

Definition 3. Structural congruence, written $\equiv$ over the set of simple processes $\mathcal{P}$ is the smallest congruence that allows the reorder of terms in the choice. 
We use structural congruence as a relation to talk about individual terms in the summation, and to to avoid to be bothered by the order of terms in the summation. For example $(a, \lambda) \cdot \mathrm{E}_{1}+(b, \mu) \cdot \mathrm{E}_{2} \equiv(b, \mu) \cdot \mathrm{E}_{2}+(a, \lambda) \cdot \mathrm{E}_{1}$. We use $S$ to indicate a set of terms of the summation that we do not wish to identfy i.e. $(a, \lambda) \cdot \mathrm{E}_{1}+(b, \mu) \cdot \mathrm{E}_{2}+(c, \gamma) \cdot \mathrm{E}_{3} \equiv(a, \lambda) \cdot \mathrm{E}_{1}+S$.

Differently from CTMC, not all transitions in SSPA have a real number attached. These are called passive transitions. Passive transitions are transitions whose delay has not yet been specified. The difference between passive and active transitions, is crucial in this work, so we proceed now to define such entities via analysis of the labels in a process.

Definition 4. A label $a \in$ Act is called active with respect to a simple process $\mathrm{M}$ if $\mathrm{M} \equiv(a, \lambda) \cdot \mathrm{M}^{\prime}+S$. A label $a \in$ Act is called passive with respect to a simple process $\mathrm{M}$ if $\mathrm{M} \equiv\left(a, x_{a}\right) \cdot \mathrm{M}^{\prime}+S$.

We now define a the set of labels that are active or passive in any possible evolution of the simple process.

Definition 5. The set of active labels, written $\mathcal{A}(\mathrm{M})$, is recursively defined as follows:

(Nil) $\mathcal{A}(0)=\emptyset$;

(Def) $\mathcal{A}(\mathrm{D})=\mathcal{A}(\mathrm{M}) \quad$ if $\mathrm{D} \stackrel{d f}{=} \mathrm{M}$;

(Choice) $\mathcal{A}\left(\sum_{i \in I}\left(a_{i}, r_{i}\right) . \mathrm{E}_{i}\right)=\cup_{i \in I}\left\{a_{i}:\left(a_{i}, r_{i}\right) . E_{i}, r_{i} \in \mathbb{R}^{+}\right\} \cup \mathcal{A}\left(\mathrm{E}_{i}\right)$;

(Closure) $\mathcal{A}\left(\mathrm{M}_{[a \leftarrow \lambda]}\right)=\mathcal{A}(\mathrm{M}) \cup\{a\}$.

Definition 6. The set of passive labels, written $\mathcal{P}(\mathrm{M})$, is recursively defined as follows:

(Nil) $\mathcal{P}(0)=\emptyset$;

(Def) $\mathcal{P}(\mathrm{D})=\mathcal{P}(\mathrm{M}) \quad$ if $\mathrm{D} \stackrel{d f}{=} \mathrm{M}$;

(Choice) $\mathcal{P}\left(\sum_{i \in I}\left(a_{i}, r_{i}\right) . \mathrm{E}_{i}\right)=\cup_{i \in I}\left\{a_{i}:\left(a_{i}, r_{i}\right) . E_{i}, r_{i} \in \operatorname{Var}\right\} \cup \mathcal{P}\left(\mathrm{E}_{i}\right)$;

(Closure) $\mathcal{P}\left(\mathrm{M}_{[a \leftarrow \lambda]}\right)=\mathcal{P}(\mathrm{M}) \backslash\{a\}$.

We simply write $\mathcal{P}$ and $\mathcal{A}$ for the set of passive and active labels when it is clear from the context which simple process we are referring to.

Definition 7. A simple process $\mathrm{M}$ is closed if $\mathcal{A}(\mathrm{M})=\emptyset$, it is open otherwise.

The closure operator transforms each open automata into a closed one. We now present a few properties of the closure operator, with respect to the semantics equivalence of strong bisimilarity.

Definition 8. We define strong bisimilarity as the largest symmetrical relation $\cong$ such that if $\mathrm{M}_{1} \cong \mathrm{M}_{2}$, then if for all $\mathrm{M}_{1}^{\prime}$ it holds that $\mathrm{M}_{1} \stackrel{a, r}{\longrightarrow} \mathrm{M}_{1}^{\prime}$ then there exists $\mathrm{M}_{2}^{\prime}$ such that $\mathrm{M}_{2} \stackrel{a, r}{\longrightarrow} \mathrm{M}_{2}^{\prime}$ and $\mathrm{M}_{1}^{\prime} \cong \mathrm{M}_{2}^{\prime}$.

Proposition 1. 1. $\mathrm{M}_{[a \leftarrow \lambda]} \cong \mathrm{M}$ if $\mathrm{M}$ is closed.

2. $\mathrm{M}_{[a \leftarrow \lambda][b \leftarrow \mu]} \cong \mathrm{M}_{b \leftarrow \mu][a \leftarrow \lambda]}$. 
3. Let $\mathcal{P}(\mathrm{M}) \cong\left\{a_{1}, a_{2}, \ldots a_{N}\right\}$ be the set of passive actions of $\mathrm{M} \cdot \mathrm{M}_{[} a_{1} \leftarrow$ $\left.\lambda_{1}\right] \ldots\left[a_{N} \leftarrow \lambda_{N}\right]$ is closed.

Proof. By induction on grammar of simple processes.

Sometimes, in the presence of multiple applications of the closure operator, we write $\mathrm{M}_{\mathcal{P} \leftarrow R}$, where $R$ is a set of rates $R=\left\{r_{1}, r_{2}, \ldots, r_{N}\right\}$ and $\mathcal{P}=$ $\left\{a_{1}, a_{2}, \ldots, a_{N}\right\}$ is the set of passive labels in M. Clearly, by generalisation of Proposition 1 this abbreviation is well defined, as it does not matter the order in which the closure is performed. Now we define the interaction among simple

$$
\begin{array}{cc}
\frac{\sum_{i \in I}\left(a_{i}, r_{i}\right) \cdot \mathrm{E}_{i} \stackrel{a_{i}, r_{i}}{\longrightarrow} \mathrm{E}_{i}}{\left(a, x_{a}\right) \cdot \mathrm{E} \stackrel{a, x_{a}}{\longrightarrow} \mathrm{E}} & \frac{\mathrm{E} \stackrel{a, r}{\longrightarrow} \mathrm{E}^{\prime}}{D \stackrel{a, r}{\longrightarrow} \mathrm{E}^{\prime}} \text { if } D \stackrel{d f}{=} \mathrm{E} \\
\frac{(a, \mu) \cdot \mathrm{E} \stackrel{a, \mu}{\longrightarrow} \mathrm{E}}{\left(a, x_{a}\right) \cdot \mathrm{E}_{[a \leftarrow \lambda]} \stackrel{a, \lambda}{\longrightarrow} \mathrm{E}_{[a \leftarrow \lambda]}} & \frac{(a, \mu) \cdot \mathrm{E}_{[a \leftarrow \lambda]} \stackrel{a, \mu}{\longrightarrow} \mathrm{E}_{[a \leftarrow \lambda]}}{}
\end{array}
$$

Table 1. Transition semantics of simple processes

$$
\begin{aligned}
& \frac{\mathrm{M}_{i} \stackrel{a, r}{\longrightarrow} \mathrm{M}_{i}^{\prime}}{\bigotimes_{L}\left(\mathrm{M}_{1}, . ., \mathrm{M}_{i}, . . \mathrm{M}_{n}\right) \stackrel{a, r}{\longrightarrow} \bigotimes_{L}\left(\mathrm{M}_{1}, . ., \mathrm{M}_{i}^{\prime}, . . \mathrm{M}_{n}\right)} \quad(a \notin L) \\
& \mathrm{M}_{i} \stackrel{a, \lambda}{\longrightarrow} \mathrm{M}_{i}^{\prime} \quad \mathrm{M}_{k} \stackrel{a, x_{a}}{\longrightarrow} \mathrm{M}_{k}^{\prime} \\
& \bigotimes_{L}\left(\mathrm{M}_{1}, . ., \mathrm{M}_{i}, . . \mathrm{M}_{k}, . . \mathrm{M}_{n}\right) \stackrel{a, \lambda}{\longrightarrow} \bigotimes_{L}\left(\mathrm{M}_{1}, . ., \mathrm{M}_{i}^{\prime}, . ., \mathrm{M}_{k}^{\prime}, . . \mathrm{M}_{n}\right) \\
& (a \in L, k \neq i)
\end{aligned}
$$

Table 2. Transition semantics of interacting processes

processes.

Definition 9. The set of interacting processes, $\mathcal{L}$, is defined by the following syntax:

$$
\mathrm{C}::=\mathrm{M} \mid \bigotimes_{L}\left(\mathrm{M}_{1}, \ldots, \mathrm{M}_{N}\right)
$$

where $L \subseteq$ Act, $\mathrm{M}$ was defined in Definition 1 , and for all $i, j \leq N$ if $i \neq j$ it holds that $\mathcal{A}\left(\mathrm{M}_{i}\right) \cap \mathcal{A}\left(\mathrm{M}_{j}\right) \cap L=\mathcal{P}\left(\mathrm{M}_{i}\right) \cap \mathcal{P}\left(\mathrm{M}_{j}\right) \cap L=\emptyset$.

Definition 10. The labelled transition system for the interacting processes written $\rightarrow: \mathcal{L} \times\left(\right.$ Act $\left.\times \mathbb{R}^{+} \cup \operatorname{Var}\right) \times \mathcal{L}$ is the smallest multi-relation that satisfies the rules in Table 1 . 
$\rightarrow$ We write $\mathrm{C} \stackrel{a, r}{\longrightarrow} \mathrm{C}^{\prime}$ if $\left(\mathrm{C},(a, r), \mathrm{C}^{\prime}\right) \in \rightarrow$, and $\rightarrow^{*}$ for the transitive closure of

Crucial to this definition is that the interaction happens pairwise. For example, in queueing networks such as the Jackson network [5], this captures the idea that customers hop from one node to one other.

Comparison with PEPA The semantics of the interaction in SSPA has been inspired by PEPA [10], but it is not identical. PEPA's interaction operation works on broadcasting while in SSPA the interaction/cooperation is strictly pairwise.

In PEPA, cooperating processes over the same set of actions $L$ is commutative and associative with the respect to a notion of strong bisimulation. Strong bisimilarity $(\cong)$ identifies processes that can carry out the same transitions with respect to the transition relation defined in Definition 10. Therefore, we assume that Definition 8 is adapted to the interacting processes.

In SSPA, the cooperating operator is commutative, but not associative with respect to strong bisimilarity, even under the same set of cooperating actions. Commutativity says that the order in which processes cooperate does not matter. If fact, two processes that differ only for the order of simple processes in the cooperation are strongly bisimilar, and, we will see, they have the same productform solution. However, as far associativity goes, the reader can verify that $\left(\mathrm{M}_{1} \oplus_{L} \mathrm{M}_{2}\right) \oplus_{L} \mathrm{M}_{3}$ and $\mathrm{M}_{1} \oplus_{L}\left(\mathrm{M}_{2} \oplus_{L} \mathrm{M}_{3}\right)$ have different transitions i.e they are not strongly bisimilar. To see this it suffices to take the following processes $\left(\mathrm{M}_{1}=\right.$ $(a, \lambda) .0$ and $\mathrm{M}_{2}=\mathrm{M}_{3}=\left(a, x_{a}\right) .0$ with $L=\{a\}$ and verify that $\left(\mathrm{M}_{2} \oplus_{L} \mathrm{M}_{3}\right)$ has no transition according to the semantics of SSPA. Therefore $M_{1} \oplus_{L}\left(M_{2} \oplus_{L} M_{3}\right) \cong 0$ while $\left(M_{1} \oplus_{L} M_{2}\right) \oplus_{L} M_{3} \neq 0$, which implies, differently from PEPA semantics, that $\left(\mathrm{M}_{1} \oplus_{L} \mathrm{M}_{2}\right) \oplus_{L} \mathrm{M}_{3} \neq \mathrm{M}_{1} \oplus_{L}\left(\mathrm{M}_{2} \oplus_{L} \mathrm{M}_{3}\right)$. If we had used PEPA transition system we would have been able to prove that the two processes are strongly bisimilar.

\section{$3.1 \quad$ CTMC}

In this paper, we deal only with product-form solutions for time-homogenous CTMCs. For a time-homogenous CTMC, the generator matrix $\mathbf{Q}$ contains all the information to compute the transient and steady-state probability distribution. From the matrix $\mathbf{Q}$ it is possible to describe the state space of the CTMC and vice versa. Generally, in process algebra such as PEPA, the language is a means to describe in a modular way the state space of the underlying CTMC. The generator matrix is then appropriately recovered for computation purposes. If the interacting process $C$ does not contain passive transitions, we can recover the CTMC by taking the set of all derivatives of $C$ as the state space of the CTMC, and by generating the entries of the generator matrix $\mathbf{Q}_{\mathrm{C}}$ as the sum of all the real numbers of the transitions between two derivatives. Rates of self-loops should be ignored, and the diagonal of the generator matrix $\mathbf{Q}_{\mathrm{C}}$ is constructed as usual to ensure that the sum of the entries of the rows equals 0. Even in 
the presence of passive transitions in a process, the generator matrix can be recovered. However, the matrix may not be used for computation purposes, as it may contain the variables from the passive transitions. For this reason, in what follows, we describe how to build the generator matrix, and we will leave it to the reader, or to the context in which it is used, to establish if the generator can be used straightforwardly for computation purposes, or instantiation of variables is necessary.

Given a process, we define the set of derivatives as the set of processes derived via the transitive closure of the labelled transition system.

The set of derivatives of an interactive process $C$ is defined as $\mathcal{S}_{\mathrm{C}}=\left\{\mathrm{C}^{\prime}\right.$ : $\left.\mathrm{C} \stackrel{a, r}{\longrightarrow}{ }^{*} \mathrm{C}^{\prime}\right\}$. The transition rate from the state of the chain $\mathrm{C}$ to $\mathrm{C}^{\prime}$ is given by the sum of the rates of all the labelled transitions of the process i.e.:

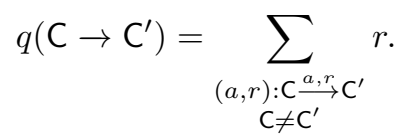

If all transition rates $q\left(C \rightarrow C^{\prime}\right) \in \mathbb{R}^{+}$then $\mathbf{Q}_{\mathbf{C}}$ is the generator matrix of the underlying CTMC of the interactive process. If for some rates it holds that $q\left(\mathrm{C} \rightarrow \mathrm{C}^{\prime}\right) \notin \mathbb{R}^{+}$then we must specify that the variables in the prefix $(a, x)$. $\mathrm{E}$ are considered the same if they occur with the same label. This observation has a huge impact in correct derivation of the generator matrix. For all intents and purposes, variables are considered the same if they are associated with the same label. For example we could write $\mathrm{M}=(a, x) \cdot(b, \mu) \cdot \mathrm{M}+(a, y) \cdot(c, z) \cdot \mathrm{M}$, however, in the construction of the generator matrix, either $x$ or $y$ will appear in the definition of the rate. This concept has no meaning from the point of view of the process algebra, but it has huge impact in the building of the generator matrix, and in the computation of probabilities. The generator matrix of $M$, written $Q_{M}$, will be

$$
\mathbf{Q}_{\mathbf{M}}=\left(\begin{array}{ccc}
-2 x & x & x \\
\mu & -\mu & 0 \\
0 & z & -z
\end{array}\right)
$$

while the matrix

$$
\mathbf{Q}_{\mathbf{M}}=\left(\begin{array}{ccc}
-(x+y) & x & y \\
\mu & -\mu & 0 \\
0 & z & -z
\end{array}\right)
$$

is not what we intended. We impose that $x=y$, since they appear with the label $a$ and therefore we can treat $x$ as a variable, and apply standard numerical operations. The semantics for the variables is such that $x, y \neq z$ as $z$ occurs with the label $c$, not $a$. For this reason the subscript of the label of the action in variables is used in this paper.

For convenience we write $q\left(\mathrm{C} \stackrel{a}{\rightarrow} \mathrm{C}^{\prime}\right)=\sum_{r: \mathrm{C} \stackrel{a, r}{\rightarrow} \mathrm{C}^{\prime}} r$ for the transition rate with respect to a label $a$. We note that for the latter definition we also consider rates from a state to itself i.e. $q(\mathrm{C} \stackrel{a}{\rightarrow} \mathrm{C})$. This will be useful later in Theorem 1 . We observe that $q\left(\mathrm{C} \rightarrow \mathrm{C}^{\prime}\right)=\sum_{\substack{a \in A c t \\ \mathrm{C} \neq \mathrm{C}^{\prime}}} q\left(\mathrm{C} \stackrel{a}{\rightarrow} \mathrm{C}^{\prime}\right)$. 
Given an interacting process $C$, we can generate the state space of the CTMC as $\mathcal{S}_{\mathrm{C}}$ and the generator matrix $\mathbf{Q}_{\mathrm{C}}$, then with an abuse of notation we refer to the properties of the process meaning the properties of the CTMC. Therefore, we can talk about a stationary or steady-state distribution of the process, $\pi_{\mathrm{C}}$, meaning that its CTMC has a stationary or steady-state distribution $\pi$. If $\mathbf{Q}_{\mathrm{C}}$ is the generator matrix of the underlying CTMC of C, then we write $\pi(\mathrm{C})$ for invariant measure meaning the $\pi \mathbf{Q}_{\mathrm{C}}=\mathbf{0}$. In other words, we use in the notation $\pi(\mathrm{C})$ instead of $\pi \mathbf{Q}_{\mathrm{C}}$. For $\mathrm{C}^{\prime} \in \mathcal{S}_{\mathrm{C}}$ we write $\boldsymbol{\pi}_{\mathrm{M}}\left(\mathrm{C}^{\prime}\right)$ meaning the value of the vector $\pi_{M}$ for the element $C^{\prime}$. If $\sum_{C^{\prime} \in \mathcal{S}_{C}} \pi\left(C^{\prime}\right)=1$ then the CTMC is ergodic and $\pi$ is the state-state distribution of $C^{[}[5$.

\section{Product-form solution}

We now present the main theorem of the paper regarding product-form solution for SSPA. The theorem asserts that for a given class of processes, that satisfies certain conditions on the rates and on the structure of state space, the productform solution exists. We start with the definition of structure of the processes.

Definition 11. In a simple process $\mathrm{M}=\sum_{i \in I}\left(a_{i}, r_{i}\right) . \mathrm{E}_{i}$ the label $a$ is the unique passive label if and only if $\mathrm{M} \equiv\left(a, x_{a}\right) \cdot \mathrm{E}+S$ and $\mathrm{M} \equiv\left(a, x_{a}\right) \cdot \mathrm{E}^{\prime}+S^{\prime}$ then $S^{\prime}=S$ and $\mathrm{E}=\mathrm{E}^{\prime}$.

We now define a set of unique passive labels for a process. Such a set is not empty if in all possible evolution of the process, one passive transition with a given label is possible.

Definition 12. The set of unique passive labels in a simple process $\mathrm{M}$, written $\mathcal{U}(\mathrm{M})$, is recursively defined as follows:

(Nil) $\mathcal{U}(0)=A c t$

(Def) $\mathcal{U}(\mathrm{D})=\mathcal{U}(\mathrm{M}) \quad$ if $\mathrm{D} \stackrel{d f}{=} \mathrm{M}$

(Choice) $\mathcal{U}\left(\sum_{i \in I}\left(a_{i}, r_{i}\right) . \mathrm{E}_{i}\right)= \begin{cases}\emptyset & \text { if there exist a passive } \\ & \text { label in } \sum_{i \in I}\left(a_{i}, r_{i}\right) \cdot \mathrm{E}_{i} \\ & \text { which is not unique } \\ \left(\cup_{i \in I}\left\{a_{i}\right\}\right) \cap_{i \in I} \mathcal{U}\left(\mathrm{E}_{i}\right) & \text { if } a_{i} \text { is a unique } \\ & \text { passive label in } \\ & \sum_{i \in I}\left(a_{i}, r_{i}\right) . \mathrm{E}_{i}\end{cases}$

(Closure) $\mathcal{U}\left(\mathrm{M}_{[a \leftarrow \lambda]}\right)=\mathcal{U}(\mathrm{M}) \backslash\{a\}$

Such a definition is necessary as we need to use process that have one passive transition. This restriction could be relaxed, but it would involve a more complicated statement of Theorem 1 .

We now provide the definition of well-formed simple processes, which are the building blocks for the correct definition of product form solutions. Well-formed processes are processes that will generate no confusion in the construction of 
product form solutions. Informally, we can think product-form as a way of decomposing the invariant measure of a CTMC. Now, if the CTMC has been built using simple processes and an empty cooperation set, then each simple process is independent of the other, and trivially the invariant measure can be written as the product of the invariant measures of each simple process. However, if a complex CTMC has been built using simple processes and a non-empty cooperation, then the behaviour of each simple process can be influenced by the others in the cooperation. If, however, in each simple process, the reversed rates of the cooperating transitions are constant in each state, then the invariant measure of a complex CTMC can be written as the product of the invariant measures of each simple process, in a similar fashion as if they were independent. To perform all these calculations correctly, we need to make sure that no confusion arises when writing the processes in SSPA, and therefore we need the notion of well-formed processes.

Definition 13 (Well-formed processes). A simple process $\mathrm{M}$ is well-formed if:

1. $\mathcal{A}(\mathrm{M}) \cap \mathcal{P}(\mathrm{M})=\emptyset$ and

2. if $\mathcal{P}(\mathrm{M}) \neq \emptyset$ then $\mathcal{P}(\mathrm{M})=\mathcal{U}(\mathrm{M})$.

From a syntactic point of view, we have done the work for the following result for the product-form solution. The theorem considers only complex CTMCs composed by well-formed processes. A further condition is added on the outgoing rates of the simple processes to guarantee that on average, we can quantify the dependency among the various processes.

Theorem 1. Given an interacting process $\mathrm{C}=\bigoplus_{L}\left(\mathrm{M}_{1}, \mathrm{M}_{2}, \ldots, \mathrm{M}_{N}\right)$ composed by be well-formed simple processes $\mathrm{M}_{1}, \mathrm{M}_{2}, \ldots, \mathrm{M}_{N}$ that cooperate on a finite set of actions $L=\left\{a_{1}, a_{2}, \ldots a_{M}\right\}$.

Assume that the state space of $\mathcal{S}_{\mathrm{C}}=\mathcal{S}_{\mathrm{M}_{1}} \times \mathcal{S}_{\mathrm{M}_{2}} \times \cdots \times \mathcal{S}_{\mathrm{M}_{N}}$ is irreducible. If for all labels in the cooperation set $L$ there exists a set of positive real numbers $K=\left\{\kappa_{1}, \ldots, \kappa_{M}\right\}$ such that, for any simple process $\mathbf{M}_{i}$, the following equations are satisfied

$$
\frac{\sum_{\mathrm{M}^{\prime} \in \mathcal{S}_{\mathrm{M}_{i}}} q\left(\mathrm{M}^{\prime} \stackrel{a_{r}}{\longrightarrow} \mathrm{M}\right) \pi_{i}\left(\mathrm{M}^{\prime}\right)}{\pi_{i}(\mathrm{M})}=\kappa_{r} \quad \mathrm{M} \in \mathcal{S}_{\mathrm{M}_{i}}, a_{r} \in L \cap \mathcal{A}\left(\mathrm{M}_{i}\right)
$$

where $\pi_{i}$ is the invariant measure of the closed process $\mathrm{M}_{i}^{c}=\mathrm{M}_{i[\mathcal{P} \cap L \leftarrow K]}$. Then the following statements hold:

1. The invariant measure of the process $\bigoplus_{L}\left(\mathrm{M}_{1}, \mathrm{M}_{2}, \ldots, \mathrm{M}_{N}\right)$ has the productform:

$$
\pi\left(\bigoplus_{L}\left(\mathrm{M}_{1}, \mathrm{M}_{2}, \ldots, \mathrm{M}_{N}\right)\right)=\pi_{1}\left(\mathrm{M}_{1}^{c}\right) \otimes \pi_{2}\left(\mathrm{M}_{2}^{c}\right) \otimes \ldots \otimes \pi_{2}\left(\mathrm{M}_{N}^{c}\right)
$$

where $\otimes$ is the Kronecker product 11 .

\footnotetext{
${ }^{1}$ If $\pi_{1}, \pi_{2}$ are two vectors, $\pi_{1} \in \mathbb{R}^{1 \times n}$ and $\pi_{2} \in \mathbb{R}^{1 \times m}$ then he Kronecker product is $\pi_{1} \otimes \pi_{2}=\left(p_{1} \pi_{2}, p_{2} \pi_{2}, \ldots p_{n} \pi_{2}\right) \in \mathbb{R}^{1 \times n m}$.
} 
2. If $\sum_{\mathrm{M} \in S_{\mathrm{M}_{i}}} \pi_{i}(\mathrm{M})=1(i \in[1, \ldots, N])$ then $\pi$ is the steady-state probability distribution of $\bigoplus_{L}\left(\mathrm{M}_{1}, \mathrm{M}_{2}, \ldots, \mathrm{M}_{N}\right)$.

Proof. For (1) we first show that for all states $\left(\mathrm{M}_{1}, \mathrm{M}_{2}, \ldots, \mathrm{M}_{N}\right) \in \mathcal{S}_{\mathrm{M}_{1}} \times \mathcal{S}_{\mathrm{M}_{2}} \times$ $\cdots \times \mathcal{S}_{\mathrm{M}_{N}}$ we can derive $\pi\left(\mathrm{M}_{1}, \mathrm{M}_{2}, \ldots, \mathrm{M}_{N}\right)=\prod_{i=1}^{N} \pi_{i}\left(\mathrm{M}_{i}\right)$ for $\mathrm{M}_{i} \in \mathcal{S}_{\mathrm{M}_{i}^{c}}$. Since we are considering the whole state space, the result $\pi\left(\bigoplus_{L}\left(\mathrm{M}_{1}, \mathrm{M}_{2}, \ldots, \mathrm{M}_{N}\right)\right)=$ $\pi_{1}\left(\mathrm{M}_{1}^{c}\right) \otimes \pi_{2}\left(\mathrm{M}_{2}^{c}\right) \otimes \ldots \otimes \pi_{2}\left(\mathrm{M}_{N}^{c}\right)$ follows. We show now, for $N=2$ that $\pi\left(\mathrm{M}_{1}, \mathrm{M}_{2}\right)=$ $\pi_{1}\left(\mathrm{M}_{1}\right) \pi_{2}\left(\mathrm{M}_{2}\right)$ when $\mathrm{M}_{1} \oplus_{\{a, c\}} \mathrm{M}_{2}$. Generalisation to $N$ is straightforward. We observe, that with an abuse of notation we write $M_{1}$ to indicate the simple process in the cooperation, but also the process that forms the state space of $S_{M_{1}}$. The context distinguishes between these two mathematical objects.

The global balance equations for the process $\mathrm{M}_{1}$ or $\mathrm{M}_{1}^{c}$ are the following, assuming that $\mathcal{A}\left(\mathrm{M}_{1}\right) \cap L=\{a\}$ and $\mathcal{A}\left(\mathrm{M}_{2}\right) \cap L=\{c\}$

$$
\begin{aligned}
& \pi_{\mathrm{M}_{1}^{c}}\left(\mathrm{M}_{1}\right)(\sum_{\mathrm{M}_{1}^{\prime} \in \mathrm{S}_{\mathrm{M}_{1}^{c}}} \mathbf{q}_{\mathrm{M}_{1}^{c}}\left(\mathrm{M}_{1}, a, \mathrm{M}_{1}^{\prime}\right)+\underbrace{\mathbf{q}_{\mathrm{M}_{1}^{c}}\left(\mathrm{M}_{1}, c, \mathrm{M}_{1}^{\prime}\right)}_{x_{c}}+\sum_{\substack{\mathrm{M}_{1}^{\prime} \in \mathrm{S}_{1}^{c} \\
b \neq a, c}} \mathbf{q}_{\mathrm{M}_{1}^{c}}\left(\mathrm{M}_{1}, b, \mathrm{M}_{1}^{\prime}\right))= \\
& \sum_{\mathrm{M}_{1}^{\prime} \in \mathrm{S}_{\mathrm{M}_{1}^{c}}} \mathbf{q}_{\mathrm{M}_{1}^{c}}\left(\mathrm{M}_{1}^{\prime}, a, \mathrm{M}_{1}\right) \pi_{\mathrm{M}_{1}^{c}}\left(\mathrm{M}_{1}^{\prime}\right)+\sum_{\mathrm{M}_{1}^{\prime} \in \mathrm{S}_{\mathrm{M}_{1}^{c}}} \underbrace{\mathbf{q}_{\mathrm{M}_{1}^{c}}\left(\mathrm{M}_{1}^{\prime}, c, \mathrm{M}_{1}\right)}_{x_{c}} \pi_{\mathrm{M}_{1}^{c}}\left(\mathrm{M}_{1}^{\prime}\right)+ \\
& \sum_{\substack{\mathrm{M}_{1}^{\prime} \in \mathrm{S}_{\mathrm{M}_{1}^{c}} \\
b \neq a, c}} \mathbf{q}_{\mathrm{M}_{1}^{c}}\left(\mathrm{M}_{1}^{\prime}, b, \mathrm{M}_{1}\right) \pi_{\mathrm{M}_{1}^{c}}\left(\mathrm{M}_{1}^{\prime}\right) \text {. }
\end{aligned}
$$

We have underlined the transition rates what would have a variable in $M_{1}$ but a real number in $\mathrm{M}_{1}^{c}$. By definition of well-formed simple process, we know that there is only one instance of $\mathbf{q}_{\mathrm{M}_{1}^{c}}\left(\mathrm{M}_{1}, c, \mathrm{M}_{1}^{\prime}\right)$.

For $\mathrm{M}_{2}$ or $\mathrm{M}_{2}^{c}$ the global balance equations would be similar by reverting the role of the rates of the actions $a$ and $c$.

We write now the global balance equations for the global state $\left(M_{1}, M_{2}\right)$ as follows:

$$
\begin{aligned}
& \pi\left(\left(\mathrm{M}_{1}, \mathrm{M}_{2}\right)\right)\left(\sum_{\substack{\mathrm{M}_{1}^{\prime} \in \mathrm{S}_{\mathrm{M}_{1}} \\
b \neq a, c}} \mathbf{q}\left(\left(\mathrm{M}_{1}, \mathrm{M}_{2}\right), b,\left(\mathrm{M}_{1}^{\prime}, \mathrm{M}_{2}\right)\right)+\sum_{\substack{\mathrm{M}_{2}^{\prime} \in \mathrm{S}_{\mathrm{M}_{2}} \\
b \neq a, c}} \mathbf{q}\left(\left(\mathrm{M}_{1}, \mathrm{M}_{2}\right), b,\left(\mathrm{M}_{1}, \mathrm{M}_{2}^{\prime}\right)\right)+\right. \\
& \left.\sum_{\left(\mathrm{M}_{1}^{\prime}, \mathrm{M}_{2}^{\prime}\right) \in \mathrm{S}_{\mathrm{M}_{1}} \times \mathrm{S}_{\mathrm{M}_{2}}} \mathbf{q}\left(\left(\mathrm{M}_{1}, \mathrm{M}_{2}\right), c,\left(\mathrm{M}_{1}^{\prime}, \mathrm{M}_{2}^{\prime}\right)\right)+\sum_{\left(\mathrm{M}_{1}^{\prime}, \mathrm{M}_{2}^{\prime}\right) \in \mathrm{S}_{\mathrm{M}_{1} \times \mathrm{S}_{\mathrm{M}_{2}}}} \mathbf{q}\left(\left(\mathrm{M}_{1}, \mathrm{M}_{2}\right), a,\left(\mathrm{M}_{1}^{\prime}, \mathrm{M}_{2}^{\prime}\right)\right)\right) \\
& =\sum_{\substack{\mathrm{M}_{1}^{\prime} \in \mathrm{S}_{\mathrm{M}_{1}} \\
b \neq a, c}} \mathbf{q}\left(\left(\mathrm{M}_{1}^{\prime}, \mathrm{M}_{2}\right), b,\left(\mathrm{M}_{1}, \mathrm{M}_{2}\right)\right) \pi\left(\left(\mathrm{M}_{1}^{\prime}, \mathrm{M}_{2}\right)\right)+ \\
& \sum_{\substack{\mathrm{M}_{2}^{\prime} \in \mathrm{S}_{\mathrm{M}_{2}} \\
b \neq a, c}} \mathbf{q}\left(\left(\mathrm{M}_{1}, \mathrm{M}_{2}^{\prime}\right), b,\left(\mathrm{M}_{1}, \mathrm{M}_{2}\right)\right) \pi\left(\left(\mathrm{M}_{1}, \mathrm{M}_{2}^{\prime}\right)\right)+ \\
& \sum_{\left(\mathrm{M}_{1}^{\prime}, \mathrm{M}_{2}^{\prime}\right) \in \mathrm{S}_{\mathrm{M}_{1}} \times \mathrm{S}_{\mathrm{M}_{2}}} \mathbf{q}\left(\left(\mathrm{M}_{1}^{\prime}, \mathrm{M}_{2}^{\prime}\right), a,\left(\mathrm{M}_{1}, \mathrm{M}_{2}\right)\right) \pi\left(\left(\mathrm{M}_{1}^{\prime}, \mathrm{M}_{2}^{\prime}\right)+\right.
\end{aligned}
$$




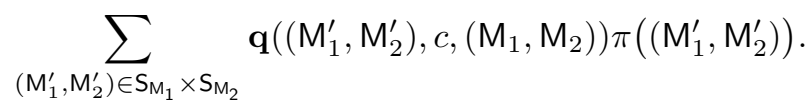

We assume that we can write the joint invariant measure in product-form, dividing by $\pi_{\mathrm{M}_{1}}\left(\mathrm{M}_{1}\right), \pi_{\mathrm{M}_{2}}\left(\mathrm{M}_{2}\right)$ and writing down the contribution of the rates of each simple process for the labels $b \notin L$ we have:

$$
\begin{aligned}
& \sum_{\substack{\mathrm{M}_{1}^{\prime} \in \mathrm{S}_{\mathrm{M}_{1}} \\
b \neq a, c}} \mathbf{q}\left(\mathrm{M}_{1}, b, \mathrm{M}_{1}^{\prime}\right)+\sum_{\substack{\left.\mathrm{M}_{1}^{\prime}, \mathrm{M}_{2}^{\prime}\right) \in \mathrm{S}_{\mathrm{M}_{1}} \times \mathrm{S}_{\mathrm{M}_{2}} \\
b \neq a, c}} \mathbf{q}\left(\left(\mathrm{M}_{1}, \mathrm{M}_{2}\right), c,\left(\mathrm{M}_{1}^{\prime}, \mathrm{M}_{2}^{\prime}\right)\right)+ \\
& =\sum_{\substack{\mathrm{M}_{1}^{\prime} \in \mathrm{S}_{\mathrm{M}_{1}} \\
b \neq a}} \mathbf{q}\left(\mathrm{M}_{1}^{\prime}, b, \mathrm{M}_{1}\right) \frac{\pi_{\mathrm{M}_{1}}\left(\mathrm{M}_{1}^{\prime}\right)}{\pi_{\mathrm{M}_{1}}\left(\mathrm{M}_{1}\right)}+\sum_{\substack{\mathrm{M}_{2}^{\prime} \in \mathrm{S}_{\mathrm{M}_{2}} \\
b \neq a, c}} \mathbf{q}\left(\mathrm{M}_{2}^{\prime}, b, \mathrm{M}_{2}\right) \frac{\pi_{\mathrm{M}_{2}}\left(\mathrm{M}_{2}^{\prime}\right)}{\pi_{\mathrm{M}_{2}}\left(\mathrm{M}_{2}\right)}+ \\
& \sum_{\left(\mathrm{M}_{1}^{\prime}, \mathrm{M}_{2}^{\prime}\right) \in \mathrm{S}_{\mathrm{M}_{1}} \times \mathrm{S}_{\mathrm{M}_{2}}} \mathbf{q}\left(\left(\mathrm{M}_{1}^{\prime}, \mathrm{M}_{2}^{\prime}\right), a,\left(\mathrm{~S}_{\mathrm{M}_{1}} \times \mathrm{S}_{\mathrm{M}_{2}}, \mathrm{M}_{2}\right)\right) \frac{\pi_{\mathrm{M}_{1}}\left(\mathrm{M}_{1}^{\prime}\right) \pi_{\mathrm{M}_{2}}\left(\mathrm{M}_{2}^{\prime}\right)}{\pi_{\mathrm{M}_{1}}\left(\mathrm{M}_{1}\right) \pi_{\mathrm{M}_{2}}\left(\mathrm{M}_{2}\right)}+ \\
& \sum_{\left(\mathrm{M}_{1}^{\prime}, \mathrm{M}_{2}^{\prime}\right) \in \mathrm{S}_{\mathrm{M}_{1}} \times \mathrm{S}_{\mathrm{M}_{2}}} \mathbf{q}\left(\left(\mathrm{M}_{1}^{\prime}, \mathrm{M}_{2}^{\prime}\right), c,\left(\mathrm{M}_{1}, \mathrm{M}_{2}\right)\right) \frac{\pi_{\mathrm{M}_{1}}\left(\mathrm{M}_{1}^{\prime}\right) \pi_{\mathrm{M}_{2}}\left(\mathrm{M}_{2}^{\prime}\right)}{\pi_{\mathrm{M}_{1}}\left(\mathrm{M}_{1}\right) \pi_{\mathrm{M}_{2}}\left(\mathrm{M}_{2}\right)}
\end{aligned}
$$

We consider the rates in the terms with joint states. We observe that since we impose that the simple processes are well formed, this means that there is only one passive action in each process: in $\mathrm{M}_{1}$ the passive action will be labelled $c$ while in $\mathrm{M}_{2}$ will be labelled $a$. The number of transitions in the joint state space $\mathrm{S}_{\mathrm{M}_{1}} \times \mathrm{S}_{\mathrm{M}_{2}}$ will the same number as the active transitions. Therefore we can rewrite the global balance equation as follows:

$$
\begin{aligned}
& \sum_{\substack{\mathrm{M}_{1}^{\prime} \in \mathrm{S}_{\mathrm{M}_{1}} \\
b \neq a, c}} \mathbf{q}\left(\mathrm{M}_{1}, b, \mathrm{M}_{1}^{\prime}\right)+\sum_{\substack{\mathrm{M}_{2}^{\prime} \in \mathrm{S}_{\mathrm{M}_{2}} \\
b \neq a, c}} \mathbf{q}\left(\mathrm{M}_{2}, b, \mathrm{M}_{2}^{\prime}\right)+\sum_{\mathrm{M}_{2}^{\prime} \in \mathrm{S}_{\mathrm{M}_{2}}} \mathbf{q}\left(\mathrm{M}_{2}, c, \mathrm{M}_{2}^{\prime}\right)+ \\
& \sum_{\mathrm{M}_{1}^{\prime} \in \mathrm{S}_{\mathrm{M}_{1}}} \mathbf{q}\left(\mathrm{M}_{1}, a, \mathrm{M}_{1}^{\prime}\right)=\sum_{\substack{\mathrm{M}_{1}^{\prime} \in \mathrm{S}_{\mathrm{M}_{1}} \\
b \neq a}} \mathbf{q}\left(\mathrm{M}_{1}^{\prime}, b, \mathrm{M}_{1}\right) \frac{\pi_{\mathrm{M}_{1}}\left(\mathrm{M}_{1}^{\prime}\right)}{\pi_{\mathrm{M}_{1}}\left(\mathrm{M}_{1}\right)}+ \\
& \sum_{\substack{\mathrm{M}_{2}^{\prime} \in \mathrm{S}_{\mathrm{M}_{2}} \\
b \neq a, c}} \mathbf{q}\left(\mathrm{M}_{2}^{\prime}, b, \mathrm{M}_{2}\right) \frac{\pi_{\mathrm{M}_{2}}\left(\mathrm{M}_{2}^{\prime}\right)}{\pi_{\mathrm{M}_{2}}\left(\mathrm{M}_{2}\right)}+\sum_{\mathrm{M}_{2}^{\prime} \in \mathrm{S}_{\mathrm{M}_{2}}} \sum_{\mathrm{M}_{1}^{\prime} \in \mathrm{S}_{\mathrm{M}_{1}}} \mathbf{q}\left(\mathrm{M}_{1}^{\prime}, a, \mathrm{M}_{1}\right) \frac{\pi_{\mathrm{M}_{1}}\left(\mathrm{M}_{1}^{\prime}\right) \pi_{\mathrm{M}_{2}}\left(\mathrm{M}_{2}^{\prime}\right)}{\pi_{\mathrm{M}_{1}}\left(\mathrm{M}_{1}\right) \pi_{\mathrm{M}_{2}}\left(\mathrm{M}_{2}\right)} \\
&+\sum_{\mathrm{M}_{1}^{\prime} \in \mathrm{S}_{\mathrm{M}_{1}}} \sum_{\mathrm{M}_{2}^{\prime} \in \mathrm{S}_{\mathrm{M}_{2}}} \mathbf{q}\left(\mathrm{M}_{2}^{\prime}, c, \mathrm{M}_{2}\right) \frac{\pi_{\mathrm{M}_{1}}\left(\mathrm{M}_{1}^{\prime}\right) \pi_{\mathrm{M}_{2}}\left(\mathrm{M}_{2}^{\prime}\right)}{\pi_{\mathrm{M}_{1}}\left(\mathrm{M}_{1}\right) \pi_{\mathrm{M}_{2}}\left(\mathrm{M}_{2}\right)} .
\end{aligned}
$$

We can now rewrite the global balance equations in Equation 3 in the following convenient way: 


$$
\begin{aligned}
\sum_{\mathrm{M}_{1}^{\prime} \in \mathrm{S}_{\mathrm{M}_{1}^{c}}} \mathbf{q}_{\mathrm{M}_{1}^{c}}\left(\mathrm{M}_{1}, a, \mathrm{M}_{1}^{\prime}\right)+\kappa_{c}+\sum_{\substack{\mathrm{M}_{1}^{\prime} \in \mathrm{S}_{\mathrm{M}_{1}^{c}} \\
b \neq a, c}} \mathbf{q}_{\mathrm{M}_{1}^{c}}\left(\mathrm{M}_{1}, b, \mathrm{M}_{1}^{\prime}\right)= \\
\left.\kappa_{a}+\sum_{\mathrm{M}_{1}^{\prime} \in \mathrm{S}_{\mathrm{M}_{1}^{c}}} \kappa_{c} \frac{\pi_{\mathrm{M}_{1}^{c}}\left(\mathrm{M}_{1}^{\prime}\right)}{\pi_{\mathrm{M}_{1}^{c}}\left(\mathrm{M}_{1}\right)}+\sum_{\substack{\mathrm{M}_{1}^{\prime} \in \mathrm{S}_{\mathrm{M}_{1}^{c}} \\
b \neq a, c}} \mathbf{q}_{\mathrm{M}_{1}^{c}}\left(\mathrm{M}_{1}^{\prime}, b, \mathrm{M}_{1}\right) \frac{\pi_{\mathrm{M}_{1}^{c}}\left(\mathrm{M}_{1}^{\prime}\right)}{\pi_{\mathrm{M}_{1}^{c}}\left(\mathrm{M}_{1}\right)}\right)
\end{aligned}
$$

By subtracting each term side of the last two equation we obtain:

$$
\begin{aligned}
\sum_{\substack{\mathrm{M}_{2}^{\prime} \in \mathrm{S}_{\mathrm{M}_{2}} \\
b \neq a, c}} \mathbf{q}\left(\mathrm{M}_{2}, b, \mathrm{M}_{2}^{\prime}\right)+ & \sum_{\mathrm{M}_{2}^{\prime} \in \mathrm{S}_{\mathrm{M}_{2}}} \mathbf{q}\left(\mathrm{M}_{2}, c, \mathrm{M}_{2}^{\prime}\right)-\kappa_{c}= \\
& \sum_{\substack{\mathrm{M}_{2}^{\prime} \in \mathrm{S}_{\mathrm{M}_{2}} \\
b \neq a, c}} \mathbf{q}\left(\mathrm{M}_{2}^{\prime}, b, \mathrm{M}_{2}\right) \frac{\pi_{\mathrm{M}_{2}}\left(\mathrm{M}_{2}^{\prime}\right)}{\pi_{\mathrm{M}_{2}}\left(\mathrm{M}_{2}\right)}+\sum_{\mathrm{M}_{2}^{\prime} \in \mathrm{S}_{\mathrm{M}_{2}}} \kappa_{a} \frac{\pi_{\mathrm{M}_{2}}\left(\mathrm{M}_{2}^{\prime}\right)}{\pi_{\mathrm{M}_{2}}\left(\mathrm{M}_{2}\right)}-\kappa_{a}
\end{aligned}
$$

The latter equation can be rewritten to see that the global balance equation of $\mathrm{M}_{2}$ by expanding the Definition of $\kappa_{a}, \kappa_{c}$ as in Condition 1 of Theorem 1 .

The proof is very elegant, not because it uses global balance equations, but because it solidly relies on the semantics of the cooperation. Such semantics establishes the contribution of each component to transform the global balance equations of the joint processes into the global balance equations of each simple process. Further considerations on the cooperation operator will lead to conclude that the semantics given in this work is the right one, as it allows the correct substitution of the rates in condition 1 of Theorem 1 in the passive transitions. As Hillston pointed out in [10, passive transitions lack of information about the speed of the transition. Such information is given by the cooperation with the active partner. In this work we embrace this view fully, but we also find out the right rates (the ones given by condition 1 of Theorem 1) for the passive transitions to proceed in isolation. We could have chosen an arbitrary rate to be substituted into the passive transition. This would have made no sense at all. The product form solution relates the rates of the joint process with the rates of the single components. We can see why it is important that cooperation is not associative, and the broadcasting semantics of PEPA would not work here. Consider three well-formed simple processes $\mathrm{M}_{1}, \mathrm{M}_{2}, \mathrm{M}_{3}$ specified as follows $\mathrm{M}_{1}=$ $(a, \lambda) \cdot \mathrm{M}_{1}^{\prime}, \mathrm{M}_{2}=\left(a, x_{a}\right) \cdot \mathrm{M}_{2}^{\prime}, \mathrm{M}_{3}=\left(a, x_{a}\right) \cdot \mathrm{M}_{3}^{\prime}$ such that condition 1 of Theorem 1 is satisfied for $\mathbf{M}_{1}$. Assume that we have PEPA semantics and $\mathrm{M}_{2} \oplus\{a\} \mathrm{M}_{3} \stackrel{a, x_{a}}{\longrightarrow}$ $\mathrm{M}_{2}^{\prime} \oplus\{a\} \mathrm{M}_{3}^{\prime}$ and $\mathrm{M}_{1} \oplus\{a\}\left(\mathrm{M}_{2} \oplus\{a\} \mathrm{M}_{3}\right) \stackrel{a, \lambda}{\longrightarrow} \mathrm{M}_{1} \oplus\{a\}\left(\mathrm{M}_{2}^{\prime} \oplus\{a\} \mathrm{M}_{3}^{\prime}\right)$. Now, to identify the product-form solution we would need to substitute in both processes the rate $\kappa_{a} \mathrm{M}_{2\left[a \leftarrow \kappa_{a}\right]}, \mathrm{M}_{3\left[a \leftarrow \kappa_{a}\right]}$. The product from would not work at all. The reader can verify this by inspecting the proof of theorem 1 Now consider the equivalent process $\left(\mathrm{M}_{1} \oplus\{a\} \mathrm{M}_{2}\right) \oplus a \mathrm{M}_{3} \stackrel{a, \lambda}{\longrightarrow}\left(\mathrm{M}_{1}^{\prime} \oplus\{a\} \mathrm{M}_{2}^{\prime}\right) \oplus\{a\} \mathrm{M}_{3}^{\prime}$ such that 
$\left(\mathrm{M}_{1} \oplus\{a\} \mathrm{M}_{2}\right) \stackrel{a, \lambda}{\longrightarrow}\left(\mathrm{M}_{1}^{\prime} \oplus\{a\} \mathrm{M}_{2}^{\prime}\right)$. We would obtain a series of substitutions $\mathrm{M}_{2\left[a \leftarrow \kappa_{a}\right]}$ and, if $\left(\mathrm{M}_{1} \oplus\{a\} \mathrm{M}_{2}\right)$ satisfy the conditions in theorem 1 for $a$, then we would have $\mathrm{M}_{3\left[a \leftarrow \kappa_{a}^{*}\right]}$, where $\kappa_{a}^{*}=\sum_{\left(\mathrm{M}_{1}^{*}, \mathrm{M}_{2}^{*}\right)} \lambda \frac{\pi\left(\mathrm{M}_{1}^{*}, \mathrm{M}_{2}^{*}\right)}{\pi\left(\mathrm{M}_{1}^{\prime \prime}, \mathrm{M}_{2}^{\prime \prime}\right)}$ Clearly this would lead to different product-form solution from $\mathrm{M}_{1} \oplus\{a\}\left(\mathrm{M}_{2} \oplus\{a\} \mathrm{M}_{3}\right)$. In conclusion, differently from PEPA semantics, we do not wish to have associativity as the rates used for the closure of each simple process matters. Such rates depend on how we group simple processes together. The semantics of the cooperation is the exactly was is needed to correctly interpret product-form solutions. In this work we are not concerned about numerical or analytical methods for solution equations in the form of 1 . Such methods can be found in [5].

\section{Product-form solutions for biological systems}

As stated in the introduction, product-form solutions have been mostly used in queueing theory. There has been a recent interest in product-form solution for biological system [15|1. In particular in [15|1] consider only chemical reactions, while we show here a variation of the product-form solutions for more complex systems.

Assume we have a cancerous cell, that grows, in a limited way provided that there is enough energy. In absence of energy the cell could die, with a certain probability $p$. We model the cell as follows:

$$
\begin{aligned}
\mathrm{C}_{0}= & \left(a, x_{a}\right) \cdot \mathrm{C}_{1} \\
\mathrm{C}_{1}= & \left(a, x_{a}\right) \cdot \mathrm{C}_{2}+\left(c, \gamma_{1}\right) \cdot \mathrm{C}_{0}+\left(c, \kappa_{c}\right) \cdot \mathrm{C}_{1} \\
\vdots & \vdots \\
\mathrm{C}_{N}= & \left(a, x_{a}\right) \cdot \mathrm{C}_{N}+\left(c, \gamma_{N}\right) \cdot \mathrm{C}_{0}+\left(c, \kappa_{c}\right) \cdot \mathrm{C}_{N} .
\end{aligned}
$$

The transitions labelled $a$ stand for the energy that will allow the cell to grow. As energy is provided by the environment, we model it as a passive transition. We note that the cell has a finite growth: even in the presence of an infinite amount of energy, the cell will stop growing. The transitions labelled $c$ stands for cancerous events: they could inhibit growth and kill the cell.

We model the energy as a switch, either there is energy for the cell to grow, or there is no energy:

$$
\begin{aligned}
& \mathrm{E}_{0}=(a, \lambda) \cdot \mathrm{E}_{1}+(a, \delta) \cdot \mathrm{E}_{0} \\
& \mathrm{E}_{1}=(d, \delta) \cdot \mathrm{E}_{0} .
\end{aligned}
$$

The rate $\delta$ represents the speed at which the environment supplies energy. Similarly, we model the trigger for cancer which reduces the size of the cell as a switch:

$$
\begin{aligned}
& \mathrm{T}_{0}=\left(c, x_{c}\right) \cdot \mathrm{T}_{1}+\left(c, x_{c}\right) \cdot \mathrm{T}_{0} \\
& \mathrm{~T}_{1}=(e, \nu) \cdot \mathrm{T}_{0} .
\end{aligned}
$$

f The system is the following: $\oplus_{\{a, c\}}\left(\mathrm{E}_{0}, \mathrm{C}_{0}, \mathrm{~T}_{0}\right)$, and the steady state probabilities are $\pi\left(\oplus_{a, c}\left(\mathrm{E}_{0}, \mathrm{C}_{0}, \mathrm{~T}_{0}\right)\right)=\pi_{1}\left(\mathrm{E}_{0}\right) \otimes \pi_{2}\left(\mathrm{C}_{0[a \leftarrow \delta]}\right) \otimes \pi_{3}\left(\mathrm{~T}_{0\left[c \leftarrow \kappa_{c}\right]}\right)$ where 
$\kappa_{c}=\frac{\gamma_{3} \pi_{1}\left(C_{3}\right)+\gamma_{2} \pi_{1}\left(C_{2}\right)+\gamma_{1} \pi_{1}\left(C_{1}\right)}{\pi_{1}\left(C_{0}\right)}$. We observe that $E_{0}, C_{0}, T_{0}$ are well formed process and that the conditions of Theorem 1 are satisfied.

The transition graphs of the state-space processes can be found in Figure 5.
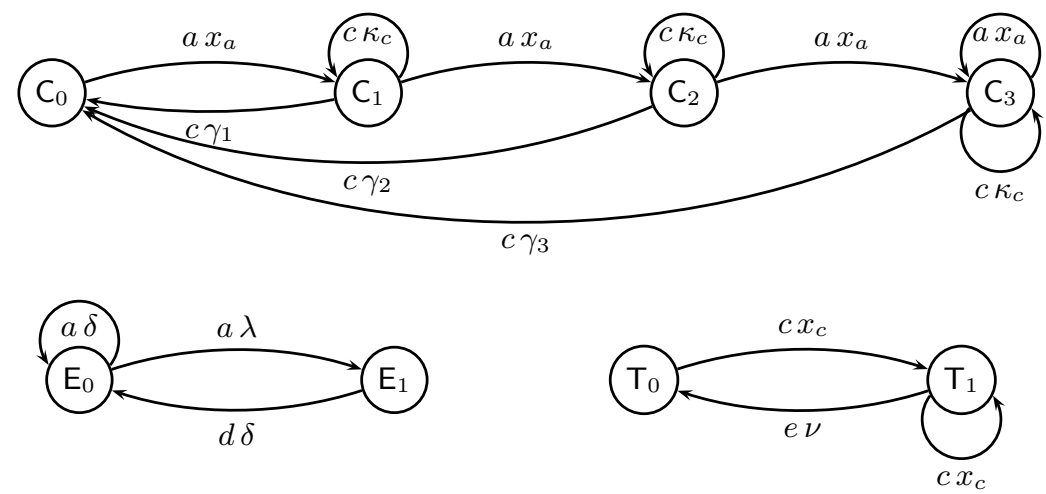

Fig. 3. Transition graphs of the state-space of the processes $E_{0}, C_{0}, T_{0}$

\section{Conclusion}

The main interest for product-form solutions arises when a CTMC contains a rather large state space, and the analytical computation of the steady state probability/invariant measure can be computationally prohibitive. In this paper, we have analysed the cooperation operator for a simple process algebra and its relationship with product-form solution. We have clarified the semantics of such operator, and we have shown that such semantics is necessary to derive correctly product-form solutions.

\section{Acknowledgments}

I gratefully acknowledge Jane Hillston for useful discussions on product-form solutions and operational semantics. Jane pointed out some mistakes and typos in earlier version of the proof of theorem 1. A lot of generous support and encouragement was given by Gianfranco Balbo. I had very interesting discussions with Andrea Marin, who also pointed out to the work done by Mairesse on biological systems. My interest in product-form solution has arisen during research work I conducted with Peter Harrison. His unique way of working has been great inspiration for me. 


\section{References}

1. David Anderson, Gheorghe Craciun, and Thomas Kurtz. Product-form stationary distributions for deficiency zero chemical reaction networks. Bulletin of Mathematical Biology, 72:1947-1970, 2010.

2. G. Balbo, S. C. Bruell, and M. Sereno. Product form solution for Generalized Stochastic Petri Nets. IEEE Trans. on Software Eng., 28:915-932, 2002.

3. G. Balbo, S. C. Bruell, and M. Sereno. On the relations between BCMP Queueing Networks and Product Form Solution Stochastic Petri Nets. Proc. of 10th Int. Workshop on Petri Nets and Performance Models, 2003., pages 103-112, 2003.

4. R. J. Boucherie. A characterisation of independence for competing Markov chains with applications to stochastic Petri nets. IEEE Tran. on Software Eng., 20(7):536544, 1994.

5. Xiuli Chao, Masakiyo Miyazawa, and Micheal Pinedo. Queueing Networks. Jon Wiley \& Sons Ltd, 1999.

6. G. Clark and J. Hillston. Product form solution for an insensitive stochastic process algebra structure. Performance Evaluation, 50:129-151, 2002.

7. J. L. Coleman, W. Henderson, and P. G. Taylor. Product form equilibrium distributions and a convolution algorithm for Stochastic Petri nets. Perform. Eval., Elsevier, 26:159-180, 1996.

8. P. Harrison and J. Hillston. Exploiting quasi-reversible structures in Markovian process algebra models. The Computer Journal, 38(7):510-520, 1995.

9. P. G. Harrison. Turning back time in Markovian process algebra. Theoretical Computer Science, 290(3):1947-1986, January 2003.

10. J. Hillston. A Compositional Approach to Perfomance Modelling. PhD thesis, Department of Computer Science, Edinburgh, 1994.

11. J. Hillston and N. Thomas. Product Form Solution for a class of PEPA Models. In Proceedings of IEEE International Computer Performance and Dependability Symposium, Durham, NC, September 1998. An extended version appeared in Performance Evaluation, 35(3-4), 1999).

12. J. Hillston and N. Thomas. Product form solution for a class of PEPA models. Perform. Eval., Elsevier, 35(3-4):171-192, 1999.

13. Jane Hillston. A class of PEPA models exhibiting product form solution over submodels. Technical Report Technical Report ECS-LFCS-98-382, University of Edingburgh, February 1998.

14. F.P. Kelly. Reversibility and Stochastic Networks. Wiley, 1979.

15. Jean Mairesse and Hoang-Thach Nguyen. Deficiency zero petri nets and product form. In Applications and Theory of Petri Nets, volume 5606 of Lecture Notes in Computer Science, pages 103-122. Springer Berlin / Heidelberg, 2009.

16. Andrea Marin and Maria G. Vigliotti. A general result for deriving productform solutions in Markovian models. In Proceedings of First Joint WOSP/SIPEW International Conference on Performance Engineering, January 2010.

17. R. Milner. Communicating and Mobile Systems: the $\pi$-calculus. Cambridge University Press, 1999.

18. Thomas G. Robertazzi. Computer Networks and Systems. Springer \& Verlag, 1994.

19. Matteo Sereno. Towards a product form solution for stochastic process algebras. The Computer Journal, 38(7):622-632, December 1995. 